\title{
Advances in the understanding and treatment of pain and headache
}

\author{
Radhouane Dallel ${ }^{1}$
}

Published online: 14 March 2020

(c) Springer-Verlag GmbH Austria, part of Springer Nature 2020

This special issue brings together leading experts in basic and clinical research on pain. The 19 manuscripts they provide give a comprehensive viewpoint on pivotal topics in pain and headache research. They address several salient issues regarding the nociceptive system, from peripheral sensory neurons, spinal (SDH) and medullary dorsal horn (MDH) circuits, cortical networks to the descending pathways that modulate the transfer of nociceptive information. They also address the pathological conditions leading to chronic pain syndromes, including orofacial pain, migraine headache, and diabetic peripheral neuropathic pain, all situations that show a very high degree of comorbidity with other pathological conditions such as sleep and affective disorders. Finally, this issue summarizes recently developed pharmacological and non-pharmacological options for pain treatment.

Despite the high prevalence of chronic pain and the urgent need for better treatments, much of its underlying mechanisms remain to be discovered. We still believe the finding of novel therapeutic targets and the development of better analgesics will rely on preclinical animal models, though we are aware that translating discoveries in animal models into actual pain therapies in humans is a major challenge. In this issue, Coderre and Laferrière (2020) examine the development of, and logistical and methodological issues about the use of animal models of chronic pain. They first describe the move toward mechanism-based or disease-related animal models of chronic pain that started 30 years ago. They discuss the experimental designs that have been implemented to reduce the number of animals used in chronic pain research and thus better comply with the " $3 \mathrm{R}$ " rules. They also address important issues regarding the advantages and disadvantages of repeated dose designs, within-group drug testing, incremental dosing schedules, and crossover designs. They finally raise concerns about changes that may

Radhouane Dallel

radhouane.dallel@uca.fr

1 Université Clermont Auvergne, CHU Clermont-Ferrand, Inserm, Neuro-Dol, F-63000 Clermont-Ferrand, France affect the evaluation of treatments, such as variations in the duration of multiple symptoms, development of nociceptive sensitization, use-dependent alterations in drug sensitivity, and time-dependent changes in pain processes in specific animal models.

Pain is a complex, multidimensional phenomenon, integrating sensory, cognitive, and affective components. It is thus considered as resulting from multiple interactions at various levels of the Central Nervous System (CNS). Physiological pain is initiated at the peripheral terminals of primary afferent fibers that are activated by noxious stimuli only and called nociceptors. The sensory inflow generated in nociceptors is then transmitted to the $\mathrm{SDH}$ or $\mathrm{MDH}$, where it is processed by complex circuits, involving excitatory as well as inhibitory interneurons, and finally transmitted to output neurons which project to several brain areas (Todd 2010).

Nociceptors are functionally characterized by the type of receptors and ion channels they express on their plasma membrane throughout the cell body and nerve fibers. These receptors/channels alert us about potentially damaging stimuli by detecting extreme temperature, high mechanical pressure, or injury-related chemicals. They transduce these stimuli into long-ranging electrical signals that are relayed to higher brain centers (Dubin and Patapoutian 2010). Nociceptors are also characterized by the various neuropeptides, most notably calcitonin gene-related peptide (CGRP), and other signaling molecules, such as adenosine triphosphate (ATP), nitric oxide, cytokines, and neurotrophic factors they express, and may release to signal to neighboring neurons, myelinating and non-myelinating Schwann cells, and satellite glial cells, resulting in modulation of the peripheral and central sensitizations that underlie pain states. Four articles in this issue review the molecules and signaling pathways involved in the detection, transduction, propagation, and modulation of nociceptive information from cutaneous, visceral, and meningeal nociceptors to the CNS.

Sharif-Naeini's review (2020) explores some recent findings on the role of mechanosensitive ion channels in cutaneous mechanical pain. Mechanotransduction is also pivotal to gastrointestinal-related visceral pain. Feng and Guo (2020) 
review the colorectal mechanisms converting mechanical stimuli into trains of action potentials at visceral sensory terminals to inform the CNS of colorectal mechanical pain. They highlight recent advances in our understanding of the biomechanics involved in the encoding process of colorectal mechanical stimuli and review the studies that investigated mechanosensitive ion channels in colorectal afferents. Among the four families of mammalian mechanosensitive ion channels, TRPV4, TRPC1, ASIC3, Piezo2, TREK-1, TREK-2, and TRAAK channels appear to directly contribute to mechanotransduction in colorectal afferents.

While trigeminal ganglion neurons are sensors of peripheral stimuli as dorsal root neurons are, they display differences in embryonic origins, transcription patterns, signaling pathways, and responses to antimigraine medications. Such differences between the two groups of sensory neurons may provide clues for selectively targeting CGRP in the treatment of migraine. Here, Messlinger et al. (2020) discuss the crosstalk signaling in the trigeminal ganglion between neurons and surrounding satellite glial cells, with a specific focus on migraine pathogenesis.

There is now evidence for interactions between the gut microbiota and visceral sensory neurons. In this issue, Defaye et al. (2020) review how gut microbiota interact with afferent sensory neurons function and pain, highlighting the role of the microbiota/gut/brain axis in the control of behaviors and neurological diseases. They also outline how changes in gut microbiota, known as dysbiosis, may influence painful gastrointestinal disorders and discuss direct host/microbiota interactions and indirect immune communication.

The SDH and MDH neuronal circuits are critical for processing sensory information generated by primary sensory afferents. In their review, Brewer and Baccei (2020) describe how neuronal activity regulates the maturation of the somatosensory system. They also discuss how the aberrant sensory input that is generated by peripheral injury or inflammation during a critical period of early postnatal development can produce enduring detrimental effects on pain processing. The authors outline the maturation of the neuronal pathways---from peripheral sensory neurons to central circuits---involved in the transmission of nociceptive signals and generation of pain sensation, as well as the influence of the neuroimmune system on somatosensation. They finally highlight the unique effects of neonatal tissue injury on somatosensation and the subsequent consequences in the adult.

Sensitization of SDH/MDH nociceptive circuits is a key phenomenon underlying pain conditions. It relates to maladaptive plastic changes as a result of peripheral tissue (e.g., inflammation) or nerve injury (Latremoliere and Woolf 2009). The cellular processes that contribute to central sensitization include enhanced postsynaptic responses of dorsal horn neurons to the release of excitatory neurotransmitters by primary afferents and reduced inhibitory tone. Here, Gradwell et al. (2020) focus on the role of dorsal horn inhibitory interneurons and the disruption of synaptic inhibition during the development and maintenance of neuropathic pain. They highlight not only recent advances in preclinical work but also the remarkable challenge we face to understand the role of inhibition in neuropathic pain. Experimental therapies, such as GABAergic neuron transplant, inhibition of apoptosis, and modulation of cation-chloride co-transporters, show promises. Nevertheless, a great deal of work is still needed before we can fully appreciate how injury produces detrimental changes in inhibitory signaling in the dorsal horn and how they can be reversed to treat neuropathic pain.

One consequence of reduced inhibitory tone within the $\mathrm{SDH} / \mathrm{MDH}$ is cutaneous mechanical allodynia, in which touch becomes painful. This symptom is not only highly prevalent in chronic pain but also difficult to treat. Peirs et al. (2020) provide a contemporary view of dorsal horn neuronal populations and describe current advances on how these populations may contribute to cutaneous mechanical allodynia. Recent progress in genetic and mass single-cell analysis has revealed its remarkable heterogeneity, while the multiple circuits for chronic pain have been unraveled in part, including those dedicated to cutaneous mechanical allodynia. The multiplicity and apparent redundancy of pain circuits most likely account for the high variability in the response of chronic pain patients to treatments, thus stressing the need for a careful consideration of symptoms, pain etiology, and neuronal circuitry, in preclinical investigations and clinical trials as well.

Accumulating evidence suggests that a specific class of excitatory interneurons within the SDH/MDH inner lamina II, that expresses the $\gamma$ isoform of protein kinase $\mathrm{C}(\mathrm{PKC} \gamma)$, plays a critical role in the expression of mechanical allodynia during chronic pain. Artola et al. (2020) review such evidence, as well as the main aspects of the anatomy, development, electrophysiology, inputs, outputs, and pathophysiology of PKC $\gamma$ neurons in the dorsal horn. They conclude that $\mathrm{PKC} \gamma$ neurons may be considered a relevant therapeutic target to alleviating mechanical allodynia during chronic pain.

Sensory information incoming to the SDH/MDH is subject to extensive integration and regulation by descending pathways that originate from noradrenergic and serotonergic systems predominantly. In this respect, diffuse noxious inhibitory controls (DNIC) or their human counterparts, conditioned pain modulation, are a unique form of endogenous descending inhibition that can be easily evoked and quantified in animals, as well as in humans. Here, Lockwood and Dickenson (2020) discuss the neuronal pathways and pharmacology of DNIC and conditioned pain 
modulation, and show how they change in pathological conditions, focusing on osteoarthritis.

Above the SDH/MDH, an extensive cortical network is associated with pain processing, as shown by evidence provided by human and animal studies over the last decades. This network includes the somatosensory, insular and cingulate cortices, as well as some frontal and parietal cortical areas. Coppola et al. (2020) present a comprehensive picture of the clinical, neurophysiological, and neuroimaging data underscoring the cortical processing of migraine headache and describe how the cortical modulation of the brainstem, midbrain, and thalamic pain pathways is critical for the initiation and maintenance of migraine attacks. On top of that, neuroimaging data have demonstrated that brains experiencing migraine undergo plastic changes in their micro- and macrostructures, as well as functions, which may manifest early in the life of migraine patients. Long-term potentiation (LTP), a putative cellular model for learning and memory, has now been reported in all cortical areas including the anterior cingulate cortex (ACC) and insular cortex (IC). Zhuo (2020) discusses the recent progress in understanding the role of the different forms of ACC and IC LTP, including synaptic tagging, in neuropathic pain. Their contribution to injuryinduced emotional changes is also discussed.

Four articles address the pathological conditions leading to chronic pain syndromes, including orofacial pain, migraine headache, and diabetic peripheral neuropathic pain, all situations that show a very high degree of comorbidity with visceral, musculoskeletal, and craniofacial pain syndromes and other pathological conditions, such as sleep and affective disorders.

The very definition of chronic orofacial pain (OFP) may vary. Here, Ananthan and Benoliel (2020) analyze the definition of chronic OFP in relation to the well-established and widely accepted definitions of chronic headache and chronic pain. They make a clear case for applying to OFP the criteria used to define "chronicity" in headache. Subsequently they examine how common OFP disorders may become chronic and discuss whether the term chronic OFP is of clinical importance and significance.

Secondly, Rosenberger et al. (2020) review our current knowledge on the pathophysiology of peripheral neuropathic pain, particularly painful diabetic neuropathy. Neuropathic pain is a frequent condition caused by a lesion or disease of the central or peripheral somatosensory nervous system, and diabetic neuropathy is a frequent cause of peripheral neuropathic pain. Rosenberger et al. (2020) discuss the state-of-the-art of clinical assessment, validity of diagnostic, screening tools, and recommendations for the management of diabetic neuropathic pain patients, including personalized pain management. They also propose a research agenda for translational research, including patient stratification for clinical trials, and improved preclinical models based on our current understanding of the underlying mechanisms.

Many pain conditions tend to co-occur in patients and thus may mutually influence their clinical expression. Affaitati et al (2020) summarize the most common concurrent pain conditions and analyze the major interactions that can be observed. The pathophysiology of such associations is complex and multifactorial. Among the possible processes underlying, the mutual influence of symptoms recorded in such associations is the modulation of central sensitization phenomena by nociceptive inputs involved in one or the other condition. Interestingly, effective treatment of one of the conditions can improve the symptoms of the other, implying that we need to evaluate systematically and thoroughly pain patients for a global effective management of their suffering.

Numerous cross-sectional studies show that there is a high degree of comorbidity between chronic pain and other pathological conditions, such as sleep and affective disorders. Herrero Babiloni et al. (2020) discuss the direction of the relationship between chronic pain and sleep disorders in adult and pediatric populations. They also summarize the putative factors influencing the interactions, such as endogenous pain modulation, inflammation, affects, mood, and cyclic alternating patterns of endogenous substance production. They provide directions for future studies in the area, opening up to the addition of brain imaging (e.g., fMRI), electrophysiology, and non-invasive brain stimulation techniques. In a close future, data obtained with these techniques may be processed using artificial intelligence and turn out to be pivotal for the personalized medicine management of patients facing chronic pain and sleep alterations.

Although opioids are commonly prescribed for chronic pain, they tend to be not very effective over time. Furthermore, the opioid crisis has recently called attention to the significant risks associated with these drugs. A number of non-opioid drugs, such as acetaminophen, non-steroidal anti-inflammatory drugs, tricyclic antidepressants, serotonin--norepinephrine reuptake inhibitor antidepressants, and anticonvulsants, are available as alternatives for chronic pain treatment. However, none of these appears to have reduced the need for opioids in managing chronic pain, and, importantly, they also have their own risk of adverse events. There is a need thus for safer and more effective alternatives to opioids for chronic pain management. Due to its ability to produce analgesia in various pain models, delta opioid receptor (DOPr) has recently emerged as a promising target for the development of new pain therapies. In this issue, Berthiaume et al. (2020) discuss the use of opioids in the management of pain and describe the evidence for the analgesic potency of DOPr agonists in animal pain models. Beside drugs, neurostimulation is another important strategy for pain management. Moisset et al. (2020) provide a 
literature review analysis on invasive and non-invasive neurostimulation techniques used to relieve neuropathic pain, nociplastic pain (mainly fibromyalgia), and headaches.

It is well known that many factors other than the disease itself may impact negatively on the symptoms and course of a disease. Nocebos represent the negative counterpart of placebos: whereas placebo effects are related to positive outcomes, nocebo effects are characterized by negative outcomes. There is today experimental evidence for nocebos creating symptoms and illness just from nothing. Particularly, a combination of biological, psychological, and social factors may interact with each other to generate a global painful experience. Several biochemical factors, e.g., cholecystokinin and cyclooxygenase, have been involved in this process. In their review, Benedetti et al. (2020) discuss old and new findings that illustrate and explain the interplay between biology and psychology in pain conditions.

In conclusion, I am very grateful to all the authors who agreed to share their deep knowledge on pain and headache in the frame of this special issue. As it is, the issue collects an original set of insightful opinion articles and reviews that attempt to tackle the multidimensional dimension of chronic pain. We hope that this issue will not only improve our understanding of pain and its management but also stimulate and foster a wider range of basic, clinical, and translational studies on pain and headache.

Acknowledgements This work was supported by funding from the Institut National de la Santé et de la Recherche Médicale (INSERM), Université Clermont Auvergne (UCA), Région Auvergne-RhôneAlpes, French government IDEX-ISITE initiative 16-IDEX-0001 (CAP 20-25) and CHU Clermont-Ferrand (France).

\section{References}

Affaitati G, Costantini R, Tana C, Cipollone F, Giamberardino MA (2020) Co-occurrence of pain syndromes. J Neural Transm (Vienna). https://doi.org/10.1007/s00702-019-02107-8

Ananthan S, Benoliel R (2020) Chronic Orofacial Pain. J Neural Transm (Vienna). https://doi.org/10.1007/s00702-020-02157-3

Artola A, Voisin D, Dallel R (2020) PKC $\gamma$ interneurons, a gateway to pathological pain in the dorsal horn. J Neural Transm (Vienna). https://doi.org/10.1007/s00702-020-02162-6

Benedetti F, Frisaldi E, Barbiani D, Camerone E, Shaibani A (2020) Nocebo and the contribution of psychosocial factors to the generation of pain. J Neural Transm (Vienna). https://doi.org/10.1007/ s00702-019-02104-x

Berthiaume S, Abdallah K, Blais V, Gendron L (2020) Alleviating pain with delta opioid receptor agonists: evidence from experimental models. J Neural Transm (Vienna)

Brewer CL, Baccei ML (2020) The development of pain circuits and unique effects of neonatal injury. J Neural Transm (Vienna). https ://doi.org/10.1007/s00702-019-02059-z
Coderre TJ, Laferrière A (2020) The emergence of animal models of chronic pain and logistical and methodological issues concerning their use. J Neural Transm (Vienna). https://doi.org/10.1007/ s00702-019-02103-y

Coppola G, Parisi V, Di Renzo A, Pierelli F (2020) Cortical pain processing in migraine. J Neural Transm (Vienna). https://doi. org/10.1007/s00702-019-02089-7

Defaye M, Gervason S, Altier C, Berthon JY, Ardid D, Filaire E, Carvalho FA (2020) Microbiota: a novel regulator of pain. J Neural Transm (Vienna). https://doi.org/10.1007/s00702-019-02083-Z

Dubin AE, Patapoutian A (2010) Nociceptors: the sensors of the pain pathway. J Clin Invest 120(11):3760-3772. https://doi. org/10.1172/JCI42843

Feng B, Guo T (2020) Visceral pain from colon and rectum: the mechanotransduction and biomechanics. J Neural Transm (Vienna). https://doi.org/10.1007/s00702-019-02088-8

Gradwell MA, Callister RJ, Graham BA (2020) Reviewing the case for compromised spinal inhibition in neuropathic pain. J Neural Transm (Vienna). https://doi.org/10.1007/s00702-019-02090-0

Herrero Babiloni A, De Koninck BP, Beetz G, De Beaumont L, Martel MO, Lavigne GJ (2020) Sleep and pain: recent insights, mechanisms, and future directions in the investigation of this relationship. J Neural Transm (Vienna). https://doi.org/10.1007/s0070 2-019-02067-z

Latremoliere A, Woolf CJ (2009) Central sensitization: a generator of pain hypersensitivity by central neural plasticity. J Pain 10(9):895-926. https://doi.org/10.1016/j.jpain.2009.06.012

Lockwood S, Dickenson AH (2020) What goes up must come down: insights from studies on descending controls acting on spinal pain processing. J Neural Transm (Vienna). https://doi.org/10.1007/ s00702-019-02077-x

Messlinger K, Balcziak LK, Russo AF (2020) Cross-talk signaling in the trigeminal ganglion: role of neuropeptides and other mediators. J Neural Transm (Vienna). https://doi.org/10.1007/s0070 2-020-02161-7

Moisset X, Lanteri-Minet M, Fontaine D (2020) Neurostimulation methods in the treatment of chronic pain. J Neural Transm (Vienna). https://doi.org/10.1007/s00702-019-02092-y

Peirs C, Dallel R, Todd AJ (2020) Recent advances in our understanding of the organization of dorsal horn neuron populations and their contribution to cutaneous mechanical allodynia. J Neural Transm (Vienna). https://doi.org/10.1007/s00702-020-02159-1

Rosenberger DC, Blechschmidt V, Timmerman H, Wolff A, Treede RD (2020) Challenges of neuropathic pain: focus on diabetic neuropathy. J Neural Transm (Vienna). https://doi.org/10.1007/ s00702-020-02145-7

Sharif-Naeini R (2020) Role of mechanosensitive ion channels in the sensation of pain. J Neural Transm (Vienna) (under revision)

Todd AJ (2010) Neuronal circuitry for pain processing in the dorsal horn. Nat Rev Neurosci 11(12):823-836. https://doi.org/10.1038/ nrn2947

Zhuo M (2020) Cortical plasticity as synaptic mechanism for chronic pain. J Neural Transm (Vienna). https://doi.org/10.1007/s0070 2-019-02071-3

Publisher's Note Springer Nature remains neutral with regard to jurisdictional claims in published maps and institutional affiliations. 